\title{
Investigations in massive 3D gravity
}

\author{
Antonio Accioly, ${ }^{1,2, *}$ José Helayël-Neto, ${ }^{1, \dagger}$ Jefferson Morais, ${ }^{1, \ddagger}$ Eslley Scatena, ${ }^{2, \S}$ and Rodrigo Turcati ${ }^{1, \|}$ \\ ${ }^{1}$ Laboratório de Física Experimental (LAFEX), Centro Brasileiro de Pesquisas Físicas (CBPF), \\ Rua Dr. Xavier Sigaud 150, Urca, 22290-180, Rio de Janeiro, RJ, Brazil \\ ${ }^{2}$ Instituto de Física Teórica (IFT), São Paulo State University (UNESP), \\ Rua Dr. Bento Teobaldo Ferraz, 271, Bloco II-Barra Funda, 01140-070, São Paulo, SP, Brazil
}

(Received 16 February 2011; published 3 May 2011)

\begin{abstract}
Some interesting gravitational properties of the Bergshoeff-Hohm-Townsend model (massive 3D gravity), such as the presence of a short-range gravitational force in the nonrelativistic limit and the existence of an impact-parameter-dependent gravitational deflection angle, are studied. Interestingly enough, these phenomena have no counterpart in the usual Einstein 3D gravity. In order to better understand the two aforementioned gravitational properties, they are also analyzed in the framework of 3D higher-derivative gravity with the Einstein-Hilbert term with the "wrong sign."
\end{abstract}

DOI: 10.1103/PhysRevD.83.104005

PACS numbers: $04.60 . \mathrm{Kz}$

\section{INTRODUCTION}

According to physical lore, field theories based on fourth (or higher) derivative actions entail unphysical ghost states of negative norm. Generally, in the literature the arguments for so undesirable a state of affairs have actually only been pushed forward for one particular class of such theories, viz. the hybrid ones in which there are both second- and fourth-order derivatives [1]; moreover, the discussion has only been formulated within the context of canonical quantization [2]. Nonetheless, it is remarkable that even among these hybrid theories, there exists a class of treelevel ghost-free models. At first sight, it seems that pure scalar curvature models, i.e. the fourth-order gravity systems with Lagrangian $R+\alpha R^{2}$, belong to this privileged elite. Actually, these systems are conformally equivalent to Einstein gravity with a massive scalar field [3]. In other words, despite having fourth derivatives at the metric level, these models are ultimately second order in their scalar-tensor versions. Just about two years ago, however, Bergshoeff, Hohm, and Townsend (BHT) [4-12] proposed a particular higher-derivative extension of the EinsteinHilbert (EH) action in three spacetime dimensions which is ghost-free at tree level [4]. The proof of unitarity given in [4] is based on the existence of an alternative form for the BHT action involving an auxiliary field, whose linearized version is equivalent to the standard Fierz-Pauli action, which is known to be ghost-free; incidentally, this proof was reviewed in [13]. We can also convince ourselves of the absence of ghosts in the linearized BHT model by directly performing a canonical analysis [14]. The BHT model, of course, radically violates standard folklore since

\footnotetext{
*accioly@cbpf.br

†helayel@cbpf.br

†morais@cbpf.br

\$scatena@ift.unesp.br

"turcati@cbpf.br
}

it is an example of a fourth-order system that is not pestered by ghosts.

Massive 3D gravity, which is also known as "massive new gravity," is actually a particular case of the most general three-dimensional theory obtained by augmenting planar gravity with the EH term with the "wrong sign" through the curvature-squared terms (3DHDG). The Lagrangian for this generic theory can be written as

$$
\mathcal{L}=\sqrt{g}\left(-\frac{2}{\kappa^{2}} R+\frac{\alpha}{2} R^{2}+\frac{\beta}{2} R_{\mu \nu}^{2}\right),
$$

where $\kappa^{2}=32 \pi G$, with $G$ being the 3D analog of Newton's constant, and $\alpha$ and $\beta$ are free coefficients. Note that the constants $\kappa, \alpha$, and $\beta$ have mass dimensions $[\kappa]=-\frac{1}{2}$ and $[\alpha]=[\beta]=-1$, in fundamental units. A glance at the propagator $\mathcal{P}$ of this theory, in momentum space-namely,

$$
\begin{aligned}
\mathcal{P}= & \frac{M_{2}^{2}}{k^{2}\left(k^{2}-M_{2}^{2}\right)} P^{(2)}+\frac{2 \lambda}{k^{2}} P^{(1)}-\frac{M_{0}^{2}}{k^{2}\left(k^{2}-M_{0}^{2}\right)} P^{(0-s)} \\
& -\frac{M_{0}^{2} \sqrt{2}}{k^{2}\left(k^{2}-M_{0}^{2}\right)}\left[P^{(0-s w)}+P^{(0-w s)}\right] \\
& +\frac{2\left[2 \lambda k^{2}-M_{0}^{2}(2 \lambda+1)\right]}{k^{2}\left(k^{2}-M_{0}^{2}\right)} P^{(0-w)},
\end{aligned}
$$

where $P^{(2)}, P^{(1)}, P^{(0-w)}, P^{(0-s)}, P^{(0-s w)}$, and $P^{(0-w s)}$ are the usual three-dimensional Barnes-Rivers operators [15,16], $M_{2}^{2}=\frac{4}{\beta \kappa^{2}}(>0), M_{0}^{2}=-\frac{4}{\kappa^{2}(8 \alpha+3 \beta)}(>0)$, and $\lambda$ is a gauge parameter-clearly shows that the 3DHDG system is nonunitary, even at tree level. We call attention to the fact that the above propagator is a special case concerning $N=1$ and $N=2$ supergravity in three dimensions augmented by (curvature) $)^{2}$ terms, which was calculated by Nishino and Rajpoot [17]; it can also be obtained from the propagator related to $3 \mathrm{D}$ gravity with the wrong sign enlarged by a Chern-Simons term [18-20]. 
On the other hand, if we set in Eq. (1) $\alpha=-\frac{3}{8} \beta$, with $\beta>0$, the equation at hand reduces to

$$
\mathcal{L}=\sqrt{g}\left[-\frac{2 R}{\kappa^{2}}+\frac{2}{\kappa^{2} m_{2}^{2}}\left(R_{\mu \nu}^{2}-\frac{3}{8} R^{2}\right)\right]
$$

where $m_{2}^{2}=\frac{4}{\kappa^{2} \beta}$ ( $m_{2}$ is a mass parameter), which is the Lagrangian for the BHT model. This system, as we have already commented, is a tree-level unitary system. Nonetheless, it is not clear at all whether or not this particular ratio between $\alpha$ and $\beta$ will survive renormalization at a given loop level, even at one loop; in other words, unitarity beyond tree level has to be checked [11]. In reality, most likely the BHT model is nonrenormalizable since it improves only the spin-2 projections of the propagator but not the spin-0 projection [21].

Our aim in this paper is twofold.

(1) To explore some interesting properties of this remarkable model that have no counterpart in the usual Einstein gravity in three-dimensional spacetime.

(2) To highlight these properties by carrying out a comparative study between them and the analogous ones obtained in the context of 3DHDG.

The article is organized as follows. In Sec. II it is shown that in the context of the BHT model, short-range gravitational forces are exerted on slowly moving test particles. These attractive forces are a distinguishing feature of massive 3D gravity. The analytical expression for the gravitational deflection angle of a light ray in the framework of the mentioned system is obtained in Sec. III. This angle, unlike that of Einstein 3D gravity, depends on the impact parameter. In Sec. IV a comparative study between the results found in Secs. II and III and the corresponding ones obtained via the 3DHDG system is done. This analysis greatly helps us to better comprehend the results of Secs. II and III. Finally, we present in Sec. V some comments and observations.

We employ natural units, $c=\hbar=1$, and our Minkowski metric is $\operatorname{diag}(+1,-1,-1)$. Our Ricci tensor is defined by $R_{\mu \nu}=R_{\mu \nu \lambda}^{\lambda} \equiv \partial_{\nu} \Gamma_{\mu \lambda}^{\lambda}-\partial_{\lambda} \Gamma_{\mu \nu}^{\lambda}+\ldots$

\section{INTERPARTICLE POTENTIAL}

In quantum mechanics the notion of a potential is introduced to be used in conjunction with the Schrödinger equation. In quantum field theory, in turn, the interactions are visualized as arising from the exchange of quanta. It is remarkable that these two concepts can be merged for slowly moving particles if we restrict ourselves to lowest-order processes. Indeed, according to the Born approximation the differential cross section concerning, for instance, the scattering of two scalar bosons with the same mass $m$ is given by $\left(\frac{d \sigma}{d \Omega}\right)_{\mathrm{CM}}=$ $\left|\frac{m}{4 \pi} \int e^{-i\left(\mathbf{p}^{\prime}-\mathbf{p}\right)} U(\mathbf{r}) d^{2} \mathbf{r}\right|$, where $\mathbf{p}$ and $\mathbf{p}^{\prime}$ are, respectively, the ingoing and outgoing momenta of one of the particles. On the other hand, the cross section for the interaction of the aforementioned bosons via graviton exchange can be expressed as $\left(\frac{d \sigma}{d \Omega}\right)_{\mathrm{CM}}=\left|\frac{\mathcal{M}_{\mathrm{NR}}}{16 m}\right|^{2}$, wherein $\mathcal{M}_{\mathrm{NR}}$ is the nonrelativistic limit of the Feynman amplitude for the process under discussion. As a result, the interparticle potential assumes the form

$$
U(r)=\frac{1}{4 m^{2}} \frac{1}{(2 \pi)^{2}} \int d^{2} \mathbf{k} \mathcal{M}_{\mathrm{NR}} e^{-i \mathbf{k} \cdot \mathbf{r}},
$$

where $\mathbf{k}$ is the momentum of the graviton exchanged.

Using the above equation we shall compute, in the following, the expression for the gravitational potential energy in the framework of the BHT model.

The elementary vertex for the process just described is

$$
\Gamma_{\mu \nu}\left(p, p^{\prime}\right)=\frac{1}{2} \kappa\left[p_{\mu} p_{\nu}^{\prime}+p_{\nu} p_{\mu}^{\prime}-\eta_{\mu \nu}\left(p \cdot p^{\prime}+m^{2}\right)\right],
$$

where the momenta are supposed to be incoming. The Feynman amplitude is, in turn, given by

$$
\begin{aligned}
\mathcal{M} & =\Gamma_{\mu \nu}\left(p,-p^{\prime}\right) \mathcal{P}^{\mu \nu, \alpha \beta} \Gamma_{\alpha \beta}\left(q,-q^{\prime}\right) \\
& =\frac{m_{2}^{2}}{k^{2}\left(k^{2}-m_{2}^{2}\right)} A+\frac{1}{k^{2}} B,
\end{aligned}
$$

where the propagator is given by

$$
\begin{aligned}
\mathcal{P}= & \frac{2 \lambda}{k^{2}} P^{(1)}+\frac{m_{2}^{2}}{k^{2}\left(k^{2}-m_{2}^{2}\right)} P^{(2)}+\frac{1}{k^{2}} P^{(0-s)} \\
& +\frac{\sqrt{2}}{k^{2}}\left(P^{(0-s w)}+P^{o-w s}\right)+\left(\frac{4 \lambda}{k^{2}}+\frac{2}{k^{2}}\right) P^{(0-w)},
\end{aligned}
$$

and

$$
\begin{aligned}
A= & \frac{1}{2} \kappa^{2}\left\{(p \cdot q)\left(p^{\prime} \cdot q^{\prime}\right)+\left(p \cdot q^{\prime}\right)\left(p^{\prime} \cdot q\right)\right. \\
& -\frac{1}{k^{2}}\left(q \cdot q^{\prime}\right)\left(m^{2}-p \cdot p^{\prime}\right)^{2}-\frac{1}{k^{2}}\left(p \cdot p^{\prime}\right)\left(m^{2}-q \cdot q^{\prime}\right)^{2} \\
& +\frac{1}{k^{2}}\left[(p \cdot q)-\left(p \cdot q^{\prime}\right)-\left(p^{\prime} \cdot q\right)+\left(p^{\prime} \cdot q^{\prime}\right)\right] \\
& \times\left(m^{2}-p \cdot p^{\prime}\right)\left(m^{2}-q \cdot q^{\prime}\right)-\left(p \cdot p^{\prime}\right)\left(q \cdot q^{\prime}\right) \\
& \left.+\frac{1}{k^{4}}\left(m^{2}-p \cdot p^{\prime}\right)^{2}\left(m^{2}-q \cdot q^{\prime}\right)^{2}\right\}
\end{aligned}
$$




$$
\begin{aligned}
B= & \frac{1}{2} \kappa^{2}\left[\left(p \cdot p^{\prime}\right)\left(q \cdot q^{\prime}\right)+\left(q \cdot q^{\prime}\right)\left(m^{2}-p \cdot p^{\prime}\right)\right. \\
& +\left(p \cdot p^{\prime}\right)\left(m^{2}-q \cdot q^{\prime}\right)+\frac{1}{k^{2}}\left(q \cdot q^{\prime}\right)\left(m^{2}-p \cdot p^{\prime}\right)^{2} \\
& +\frac{1}{k^{2}}\left(p \cdot p^{\prime}\right)\left(m^{2}-q \cdot q^{\prime}\right)^{2}+\left(m^{2}-q \cdot q^{\prime}\right) \\
& \times\left(m^{2}-p \cdot p^{\prime}\right)+\frac{1}{k^{2}}\left(m^{2}-q \cdot q^{\prime}\right)\left(m^{2}-p \cdot p^{\prime}\right)^{2} \\
& +\frac{1}{k^{2}}\left(m^{2}-q \cdot q^{\prime}\right)^{2}\left(m^{2}-p \cdot p^{\prime}\right) \\
& \left.+\frac{1}{k^{4}}\left(m^{2}-p \cdot p^{\prime}\right)^{2}\left(m^{2}-q \cdot q^{\prime}\right)^{2}\right]
\end{aligned}
$$

In the nonrelativistic limit Eq. (6) reduces to

$$
\mathcal{M}_{\mathrm{NR}}=-\frac{1}{2} \kappa^{2} \frac{m^{4}}{\mathbf{k}^{2}+m_{2}^{2}}
$$

Performing the appropriate integration using Eqs. (4) and (10), we come to the conclusion that the potential energy is given by

$$
U(r)=-2 m^{2} G K_{0}\left(m_{2} r\right),
$$

where $K_{0}$ is the modified Bessel function of order zero. Note that $K_{0}(x)$ behaves as $-\ln x$ at the origin and as $x^{-(1 / 2)} e^{-x}$ asymptotically.

The nonrelativistic potential, in turn, has the form

$$
V(r)=-2 m G K_{0}\left(m_{2} r\right) \text {. }
$$

At this point, some comments are in order.

(i) Like the Newtonian potential $V_{\text {Newt }}(r)=2 m G \ln \frac{r}{r_{0}}$, where $r_{0}$ is an infrared regulator, the potential concerning the BHT model has a logarithm singularity at the origin.

(ii) $V(r) \rightarrow 0$ as $r \rightarrow \infty$.

(iii) In the $m_{2} \rightarrow \infty$ limit, $V(r) \rightarrow 0$, reproducing, in this way, the potential of the usual threedimensional gravity.

On the other hand, in the nonrelativistic limit a test particle of mass $m_{\text {test }}$ moving in a weak gravitational field experiences a force $\mathbf{F}(r)=-m_{\text {test }} \nabla V(r)$. Now, taking Eq. (12) into account, we find that $\mathbf{F}(r)=F(r) \hat{\mathbf{r}}$, where

$$
F(r) \equiv-2 G m_{\text {test }} m m_{2} K_{1}\left(m_{2} r\right)
$$

wherein $K_{1}$ is the modified Bessel function of order one. Remembering that $-x K_{1}(x)$ is a negative monotonically increasing function in the range $0 \leq x<\infty$ since $\frac{d}{d x}\left[-x K_{1}(x)\right]=x K_{0}(x)$, we conclude that $\mathbf{F}(r)$ is always attractive (see Fig. 1). This short-range gravitational force does not exist in the framework of three-dimensional general relativity, being peculiar to the BHT model.

The potential energy given by Eq. (11) is, as expected, equivalent at the linearized level to that obtained via the (unitary) Pauli-Fierz model for a massive spin-2 field.

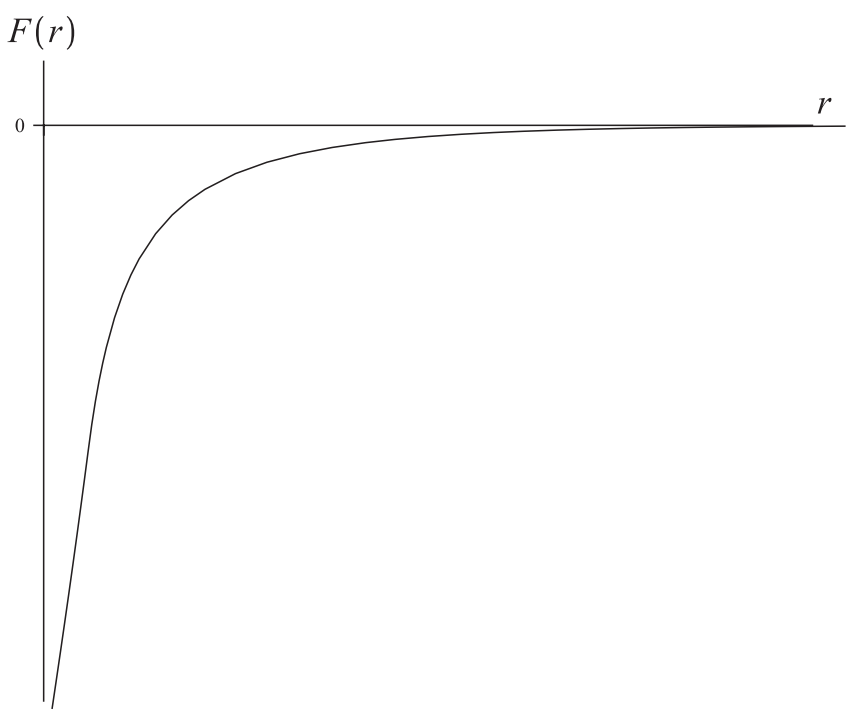

FIG. 1. The short-range gravitational force related to the BHT model.

\section{LIGHT BENDING}

Einstein 3D gravity is trivial outside the sources; consequently, we should expect, at least in principle, no light deflection in its framework. This is not so, in practice. Actually, a light ray undergoes a deflection that is proportional to the source mass; in addition, the bending is impact-parameter independent. This can be easily shown in the case of a point particle of mass $m$ located at $\mathbf{r}=\mathbf{0}$. The corresponding linearized metric, in the de Donder gauge, is given by

$$
d s^{2}=d t^{2}-(1-\lambda)\left(d r^{2}+r^{2} d \theta^{2}\right),
$$

where $\lambda=8 G m \ln \frac{r}{r_{0}}$, with $r_{0}$ being an infrared regulator, and $r$ and $\theta$ are the usual polar coordinates.

Now, introducing new radial $\left(r^{\prime}\right)$ and angular $\left(\theta^{\prime}\right)$ coordinates through the change of variables

$$
(1-\lambda) r^{2}=(1-8 G m) r^{\prime 2}, \quad \theta^{\prime}=(1-4 G m) \theta,
$$

we obtain, to linear order in $G m$,

$$
d s^{2}=d t^{2}-d r^{\prime 2}-r^{\prime 2} d \theta^{\prime 2} .
$$

Interestingly enough, the geometry around the point particle is locally identical to that of a flat spacetime; however, it is not globally Minkowskian since the angle $\theta^{\prime}$ varies in the range $0 \leq \theta^{\prime}<2 \pi(1-4 G m)$. Accordingly, the three-dimensional metric (14) describes a conical space with a wedge of angular size equal to $8 \pi G m$ removed and the two faces of the wedge identified. The light deflection angle is therefore equal to $4 \pi G m$ and is independent of the impact parameter. This phenomenon is very similar to the Aharonov-Bohm effect in electrodynamics. Indeed, there the interference pattern of two coherent electron beams moving on different sides of a thin solenoid is affected by the magnetic flux within the 
solenoid, although the magnetic field vanishes everywhere along the beams; in our case, on the other hand, the light ray propagating in the flat spacetime region around the point particle feels the spacetime curvature which is restricted to the massive point particle [22,23]. As we shall see in the following, this odd geometrical effect does not take place in massive 3D gravity. In this vein, let us compute the gravitational deflection of a light ray in the framework of the BHT model. To do that we have to solve beforehand the linearized field equations related to the BHT system.

The field equations concerning the Lagrangian density

$$
\mathcal{L}=\sqrt{g}\left[-\frac{2 R}{\kappa^{2}}+\frac{2}{\kappa^{2} m_{2}^{2}}\left(R_{\mu \nu}^{2}-\frac{3}{8} R^{2}\right)-\mathcal{L}_{\mathrm{M}}\right],
$$

where $\mathcal{L}_{\mathrm{M}}$ is the Lagrangian density for matter, are

$$
\begin{aligned}
& G_{\mu \nu}+\frac{1}{m_{2}^{2}}\left[\frac{1}{2} R_{\rho \sigma}^{2} g_{\mu \nu}-\frac{1}{4} \nabla_{\mu} \nabla_{\nu} R-2 R_{\mu \rho \lambda \nu} R^{\rho \lambda}\right. \\
& \left.\quad-\frac{1}{4} g_{\mu \nu} \square R+\square R_{\mu \nu}-\frac{3}{16} R^{2} g_{\mu \nu}+\frac{3}{4} R R_{\mu \nu}\right] \\
& =\frac{\kappa^{2}}{4} T_{\mu \nu},
\end{aligned}
$$

where $T_{\mu \nu}$ is the energy-momentum tensor, and $G_{\mu \nu} \equiv R_{\mu \nu}-\frac{1}{2} g_{\mu \nu} R$ is the Einstein tensor.

The corresponding linearized field equations are given by

$$
\begin{aligned}
& \left(1+\frac{\square}{m_{2}^{2}}\right)\left[-\frac{1}{2} \square h_{\mu \nu}+\frac{\eta_{\mu \nu}}{4 \kappa} R^{(\operatorname{lin})}\right]+\frac{1}{2}\left(\partial_{\mu} \Gamma_{\nu}+\partial_{\nu} \Gamma_{\mu}\right) \\
& =\frac{\kappa}{4}\left(\frac{T}{2} \eta_{\mu \nu}-T_{\mu \nu}\right),
\end{aligned}
$$

where $R^{(\text {lin })}=\kappa\left[\frac{1}{2} \square h-\gamma^{\mu \nu}{ }_{, \mu \nu}\right], \Gamma_{\mu} \equiv\left(1+\frac{\square}{m_{2}^{2}}\right) \partial_{\rho} \gamma_{\mu}^{\rho}+$ $\frac{\partial_{\mu} R^{\text {(in })}}{4 \kappa m_{2}^{2}}, \quad \gamma_{\mu \nu} \equiv h_{\mu \nu}-\frac{1}{2} \eta_{\mu \nu}$. Note that here indices are raised (lowered) using $\eta^{\mu \nu}\left(\eta_{\mu \nu}\right)$.

Mimicking Teyssandier's work on 4D higher-derivative gravity [24], it can be shown that it is always possible to choose a coordinate system such that the gauge conditions, $\Gamma_{\mu}=0$, on the linearized metric hold. Assuming that these conditions are satisfied, it is straightforward to show that the general solution of (17) is given by

$$
h_{\mu \nu}=\psi_{\mu \nu}-h_{\mu \nu}^{(\mathrm{E})}
$$

where $h_{\mu \nu}^{(\mathrm{E})}$ is the solution of the linearized Einstein equation in the de Donder gauge, i.e.,

$$
h_{\mu \nu}^{(\mathrm{E})}=\frac{\kappa}{2}\left(T n_{\mu \nu}-T_{\mu \nu}\right), \quad \partial^{\nu} \gamma_{\mu \nu}^{(\mathrm{E})}=0,
$$

where $\gamma_{\mu \nu}^{(\mathrm{E})} \equiv h_{\mu \nu}^{(\mathrm{E})}-\frac{1}{2} \eta_{\mu \nu} h^{(\mathrm{E})}$, while $\psi_{\mu \nu}$ satisfies the equation

$$
\left(\square+m_{2}^{2}\right) \psi_{\mu \nu}=-\frac{\kappa}{2}\left(T_{\mu \nu}-\frac{1}{2} \eta_{\mu \nu} T\right) .
$$

It is worth noticing that in this very special gauge the equations for $\psi_{\mu \nu}$ and $h_{\mu \nu}^{(\mathrm{E})}$ are totally decoupled. As a result, the general solution to Eq. (17) reduces to a linear combination of the solutions of the aforementioned equations.

Solving Eqs. (19) and (20) for a pointlike particle of mass $m$ located at $\mathbf{r}=\mathbf{0}$, we find

$$
\begin{gathered}
h_{00}=-\frac{\kappa m}{8 \pi} K_{0}\left(m_{2} r\right), \\
h_{11}=h_{22}=-\frac{\kappa m}{8 \pi}\left[K_{0}\left(m_{2} r\right)+2 \ln \frac{r}{r_{0}}\right] .
\end{gathered}
$$

Note that for $m_{2} \rightarrow \infty$, the above solution reproduces the solution of the three-dimensional linearized Einstein equation with the wrong sign, in the de Donder gauge, as it should.

We are now ready to discuss the deflection of a photon due to the gravitational field generated by the mass $m$. Suppose, in this spirit, a photon with momentum $p_{\mu}$ coming from infinity with an impact parameter $b$ (see Fig. 2).

The net change in $p_{\mu}$ while it passes through the aforementioned gravitational field is given by

$$
\Delta p_{\mu}=\frac{\kappa}{2} p^{\alpha} \int_{-\infty}^{\infty} \partial_{\mu} h_{\alpha \beta} d x^{\beta},
$$

where the integration is performed along the approximately straight line trajectory of the photon. As a consequence, the displacement along the approximately straight ray and the momentum are, respectively,

$$
d x^{\mu} \approx\left(d x^{1}, d x^{1}, 0\right), \quad p^{\mu} \approx\left(p^{1}, p^{1}, 0\right) .
$$

Inserting these quantities into Eq. (23), we obtain

$$
\Delta p_{2}=\frac{\kappa}{2} p^{1} \int_{-\infty}^{\infty} \partial_{y}\left[h_{00}+h_{11}\right] d x^{1},
$$

which can be written as

$$
\Delta p_{2}=\frac{\kappa}{2} p^{1} \int_{-\infty}^{\infty}\left\{\left[\frac{d}{d r}\left(h_{00}+h_{11}\right)\right] \frac{\partial r}{\partial y}\right\}_{y=b} d x .
$$

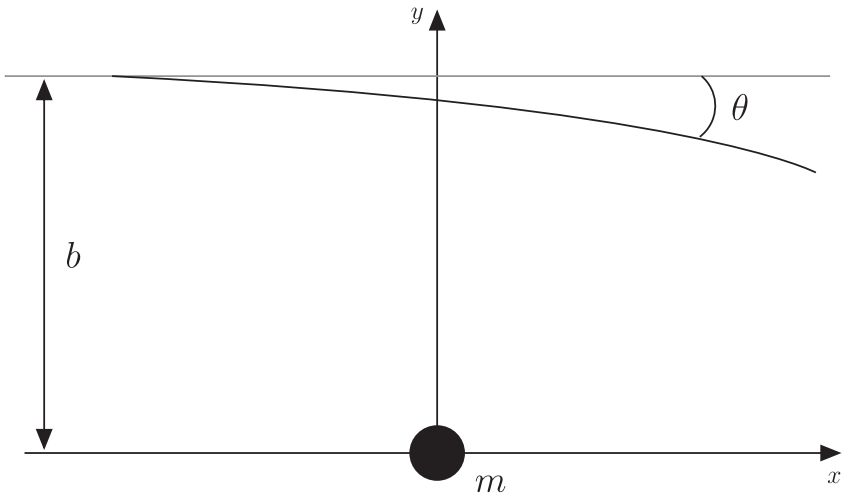

FIG. 2. Geometry of the light bending. 
With the results (21) and (22), we can write Eq. (25) simply as

$$
\Delta p_{2}=\frac{b m \kappa^{2}}{4 \pi} p^{1} \int_{0}^{\infty}\left[\frac{m_{2} K_{1}\left(m_{2} \sqrt{x^{2}+b^{2}}\right)}{\sqrt{x^{2}+b^{2}}}-\frac{1}{x^{2}+b^{2}}\right] d x .
$$

Now, taking into account that [25]

$$
\begin{aligned}
& \int K_{\nu}\left(\alpha \sqrt{x^{2}+y^{2}}\right) \frac{x^{2 \mu+1}}{\sqrt{\left(x^{2}+y^{2}\right)^{\nu}}} d x \\
& =\frac{2^{\mu} \Gamma(\mu+1)}{\alpha^{\mu+1} y^{\nu-\mu-1}} K_{\nu-\mu-1}(\alpha y), \quad \alpha>0, \quad \operatorname{Re}(\mu)>-1,
\end{aligned}
$$

and

$$
K_{+(1 / 2)}(z)=\sqrt{\frac{\pi}{2 z}} e^{-z},
$$

we promptly find

$$
\Delta p_{2}=\frac{\kappa^{2} m p^{1}}{8}\left(-1+e^{-m_{2} b}\right) .
$$

Denoting the absolute value of the deflection angle by $\theta\left(=\left|\frac{\Delta p_{y}}{p_{x}}\right|\right)$, we come to the conclusion that

$$
\theta=4 \pi G m\left(1-e^{-m_{2} b}\right) .
$$

We remark that for $m_{2} \rightarrow \infty$, the modulus of the deflection angle reduces to $4 \pi G m$, which, as we have already commented, is the geometrical prediction of 3D Einstein gravity.

\section{A COMPARATIVE ANALYSIS}

Since, as we have already discussed, the BHT model is a special case of the 3DHDG system, a comparative study between the predictions of these models concerning the nonrelativistic potential, as well as the light bending, will certainly shed a new light on the results found in Secs. II and III. Accordingly, we shall briefly analyze these phenomena in the framework of 3DHDG and carry out, afterwards, a comparative study between them and the corresponding ones obtained for the BHT system. We remark that the study of the potential energy in the context of the 3DHDG model is worthy of being done due to the astonishing similarity between this potential and that concerning the interaction of two superconducting vortices.

\section{A. The nonrelativistic potential for the 3DHDG model}

A computation similar to that of Sec. II yields the following expression for the potential energy:

$$
U(r)=2 G M^{2}\left[K_{0}\left(M_{0} r\right)-K_{0}\left(M_{2} r\right)\right] .
$$

Contrary to the Newtonian potential, $U_{\text {Newt }}(r)=$ $2 G M^{2} \ln \frac{r}{r_{0}}$, which has a logarithmic singularity at the origin and is unbounded at infinity, this potential is extremely well behaved: it is finite at the origin $(U(0)=$ $\left.2 G M^{2} \ln \frac{M_{2}}{M_{0}}\right)$ and zero at infinity. Interestingly enough, it describes three possible gravitational regimes. Indeed, depending on the choice of the parameters in the action functional, one obtains gravity $\left(M_{2}<M_{0}\right)$, antigravity $\left(M_{0}<M_{2}\right)$, or gravitational shielding $\left(M_{0}=M_{2}\right)$.

In Fig. 3 we show a schematic picture of the gravitational potential for the three situations just described. It is rather remarkable that this quantitative picture is very similar, mutatis mutandis, to the qualitative one found by Jacobs and Rebbi [26] through an approximate computer study of the two-vortex potential. In fact, evaluating the energy interaction of two vortex configurations, in the Ginzburg-Landau theory or, equivalently, in the Abelian Higgs model, as a function of the separation between the two vortices and the parameter $\lambda$, which measures the relative strength of the matter self-coupling and the electromagnetic coupling, they showed that the intervortex potential is attractive for $\lambda<1$ and repulsive for $\lambda>1$. They also found that for $\lambda=1$ a lower bound is actually reached at all separations and that, therefore, in this case vortices do not interact.

\section{B. Light bending in the context of the 3DHDG system}

A procedure analogous to that employed in Sec. III provides the general solution to the linearized field equations related to the 3DHDG model generated by a pointlike particle of mass $m$ located at $\mathbf{r}=\mathbf{0}$, i.e.,

$$
h_{00}=\frac{m \kappa}{8 \pi}\left[K_{0}\left(M_{0} r\right)-K_{0}\left(M_{2} r\right)\right],
$$

$$
h_{i i}=\frac{m \kappa}{8 \pi}\left[K_{0}\left(M_{0} r\right)-K_{0}\left(M_{2} r\right)-2 \ln \frac{r}{r_{0}}\right] .
$$

Using these results, one can then show that the absolute value of the deflection angle $\theta$ of a photon due to the gravitational field of the mass $m$ is given by

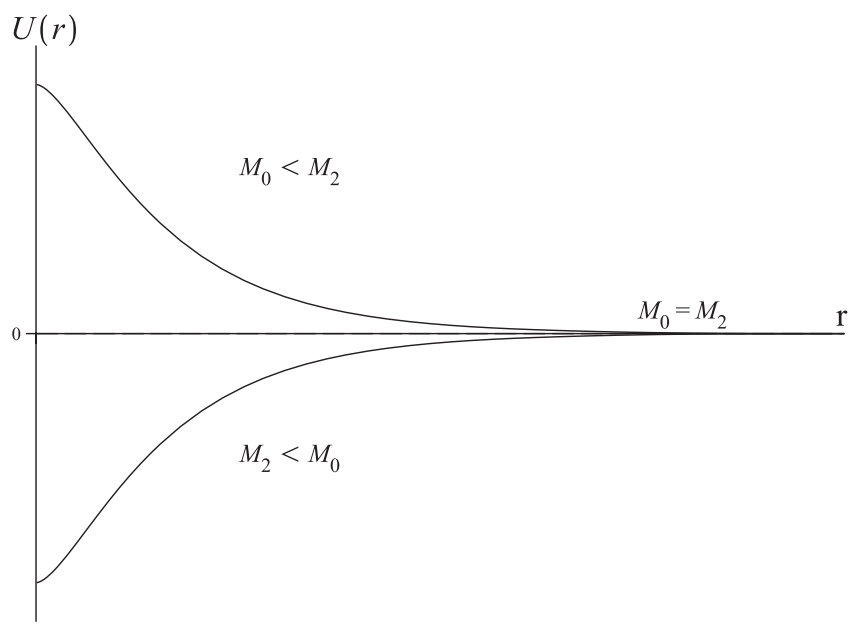

FIG. 3. The possible gravitational regimes predicted by 3DHDG. 


$$
\theta=4 \pi G m\left(1-e^{-M_{2} b}\right) .
$$

Note that the scalar excitation of mass $M_{0}$ does not contribute at all to the light bending. Why is this so? Because the metric concerning linearized $R+R^{2}$ gravity with the EH term with the wrong sign - the theory obtained by linearizing the field equations related to the action $\int d^{3} x \sqrt{g}\left[-\frac{2}{\kappa^{2}} R+\frac{\alpha}{2} R^{2}-\mathcal{L}_{\mathrm{M}}\right]$ - is conformally related to that of $3 \mathrm{D}$ linearized general relativity with the wrong sign. Indeed, denoting the solution to the linearized $R+R^{2}$ gravity with the wrong sign by $g_{\mu \nu}^{\left(R+R^{2}\right)}$, we promptly obtain

$$
\begin{aligned}
g_{\mu \nu}^{\left(R+R^{2}\right)} & \equiv \eta_{\mu \nu}+\kappa h_{\mu \nu}^{\left(R+R^{2}\right)}=(1-\kappa \phi)\left[\eta_{\mu \nu}-\kappa h_{\mu \nu}^{(\mathrm{E})}\right] \\
& =(1-\kappa \phi) g_{\mu \nu}^{(\mathrm{E})},
\end{aligned}
$$

where, of course, terms of order $\kappa^{2}$ were neglected. Here, $h_{\mu \nu}^{(\mathrm{E})}$ and $\phi$ have the same meaning as in Sec. III, while $g_{\mu \nu}^{(\mathrm{E})}$ stands for the solution of 3D linearized Einstein gravity with the wrong sign. That is the reason why the expressions (27) and (31), with the appropriate changes, are so strikingly similar.

\section{A comparative study}

We are now ready to do a comparative analysis between the two results obtained for the 3DHDG system and the corresponding ones predicted by the BHT model, so that a better physical understanding of the latter can be achieved.

From the preceding considerations, we come to the conclusion that the birth of the BHT potential is the 3DHDG one with $M_{2}<M_{0}$ (see Fig. 4). Examining the diagram depicted in Fig. 4, we clearly see that as $M_{0}$ becomes greater and greater, the 3DHDG potential rapidly approaches the BHT one and eventually the two potentials coalesce. Accordingly, to arrive at the BHT potential from the 3DHDG one, the latter must necessarily become singular at the origin, which occurs in the $M_{0} \rightarrow \infty$ limit. It is remarkable that this is precisely the condition for avoiding,

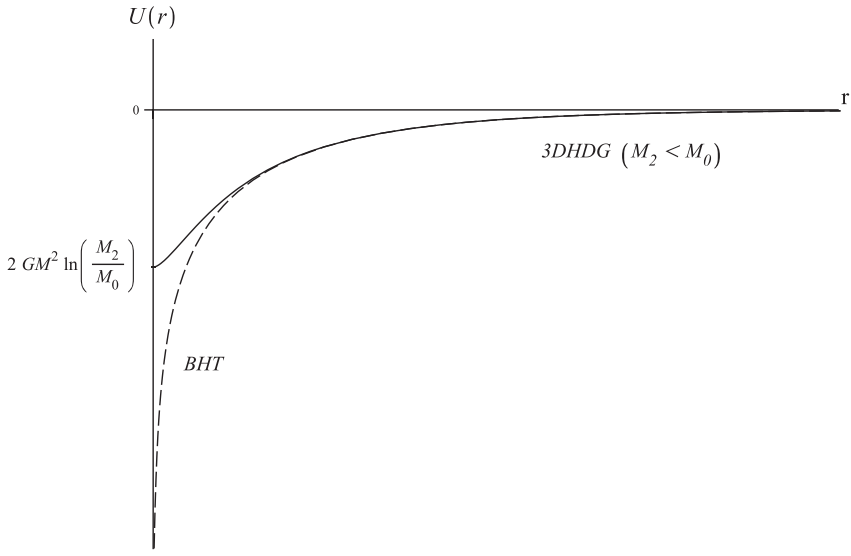

FIG. 4. Gravitational potential energy for both the 3DHDG system with $M_{2}<M_{0}$ (continuous line) and the BHT model (dashed line). at tree level, the massive spin- 0 ghost that haunts 3DHDG. Therefore, the presence of the singularity in the BHT potential is, in a sense, correlated to the absence of the tree-level ghost; in other words, unitarity at tree level and the existence of a singularity in the potential are somewhat intertwined.

We shall now improve our discussion about the light bending in the framework of the BHT model. In Sec. III we arrived at the conclusion that the gravitational deflection angle predicted by this model is impact-parameter dependent, as expected; however, is this angle small? Before answering this question, we recall that one of the reasons for doing research on three-dimensional gravity is that $3 \mathrm{D}$ gravity has a direct physical relevance to modeling phenomena that are actually dynamically confined to lower dimensionality. In fact, gravitational physics in the presence of a straight cosmic string (infinitely long, perpendicular to a plane) is adequately described by $3 \mathrm{D}$ gravity [23]. This means that the answer to our initial question can be found by analyzing the gravitational deflection in a 4D gravity model somewhat analogous to the BHT model, namely, 4D higher-derivative gravity with the wrong sign, which is defined by the Lagrangian

$$
\mathcal{L}=\sqrt{-g}\left[-\frac{2 R}{\kappa^{2}}+\frac{\alpha}{2} R^{2}+\frac{\beta}{2} R_{\mu \nu}^{2}\right] .
$$

One can show that the modulus of the deflection angle $\theta$ is now given by

$$
\theta=4 \pi G \mu\left(1-e^{-\bar{M}_{2} b}\right),
$$

where $\mu$ is the energy density of the string and $\bar{M}_{2}^{2} \equiv$ $-\frac{4}{\kappa^{2} \beta}(>0)$. If we take into account that $|\beta|=10^{60}$, we immediately get $\bar{M}_{2} \approx 10^{3} \mathrm{~cm}^{-1}[27,28]$. As a consequence, at large distances from the string the deflection angle $(\theta \approx G \mu)$ is small, as it should be. Indeed, observations constrain $G \mu \approx 10^{-5}$, while the string scenario for galaxy formation requires $G \mu \approx 10^{-6}$, corresponding to a grand-unified scale $\approx 10^{16} \mathrm{GeV}$ [23]. As a result, only for photons passing very near to the cosmic string will the light bending differ significantly from the usual value predicted by $4 \mathrm{D}$ higher-dimensional gravity with the wrong sign. The same conclusion, with the appropriate changes, applies to the BHT model.

\section{FINAL REMARKS}

As is well known, three-dimensional Einstein gravity without sources is physically vacuous because Einstein and Riemann tensors are equivalent in $D=3$. In addition, the quantization of the gravity field does not give rise to propagating gravitons since the spacetime metric is locally determined by the sources. Consequently, the description of gravitational phenomena via 3D gravity leads to some bizarre results, such as the following. 
(i) Lack of a gravity force in the nonrelativistic limit.

(ii) Gravitational deflection independent of the impact parameter.

Nevertheless, as we have discussed, these odd phenomena do not take place in the context of the BHT model. In fact, in the framework of the latter, shortrange gravitational forces are exerted on slowly moving particles; besides, the light bending depends on the impact parameter, as it should. In other words, the idiosyncrasies of $3 \mathrm{D}$ general relativity do not take place in the BHT system.

The main reason for studying 3D gravity models is, in reality, to try to find a gravity system with less austere ultraviolet divergences in perturbation theory. Since general relativity in 3D is dynamically trivial, the BHT model, which is tree-level unitary, is an important step in this direction. This kind of research conducted in lower dimensions certainly helps us to gain insight into difficult conceptional issues, which are present and more opaque in the physical $(3+1)$-dimensional world.
It is worth mentioning that the triviality of 3D general relativity can also be cured by adding to the $\mathrm{EH}$ action in 3D a parity-violating Chern-Simons term. The resulting model is commonly known as topological massive gravity (TMG) [29]. Recently, Ahmedov and Aliev [12] showed that the field equations concerning the BHT system consist of a massive (tensorial) Klein-Gordon-type equation with a curvature-squared source term and a constraint equation; moreover, for algebraic type $D$ and $N$ spacetimes, the field equations of TMG could be thought of as the "square root" of the massive Klein-Gordon-type equation, which allowed them to map all type $D$ and $N$ solutions of TMG into those of the BHT model. We remark, however, that in contrast with TMG, 3D massive gravity has the great advantage of being a parity-preserving theory.

\section{ACKNOWLEDGMENTS}

The authors are very grateful to FAPERJ, CNPq, and CAPES (Brazilian agencies) for financial support.
[1] K. Stelle, Phys. Rev. D 16, 953 (1977); Gen. Relativ. Gravit. 9, 353 (1978).

[2] P. Manheim, Found. Phys. 37, 532 (2007).

[3] B. Whitt, Phys. Lett. 145B, 176 (1984).

[4] E. Bergshoeff, O. Hohm, and P. Townsend, Phys. Rev. Lett. 102, 201301 (2009).

[5] E. Bergshoeff, O. Hohm, and P. Townsend, Phys. Rev. D 79, 124042 (2009).

[6] R. Andringa et al., Classical Quantum Gravity 27, 025010 (2010).

[7] E. Bergshoeff, O. Hohm, and P. Townsend, Ann. Phys. (N.Y.) 325, 1118 (2010).

[8] E. Bergshoeff et al., Classical Quantum Gravity 28, 015002 (2011).

[9] E. Bergshoeff et al., Classical Quantum Gravity 27, 235012 (2010).

[10] M. Nakasone and I. Oda, Prog. Theor. Phys. 121, 1389 (2009).

[11] İ. Güllü and B. Tekin, Phys. Rev. D 80, 064033 (2009).

[12] H. Ahmedov and A. Aliev, Phys. Rev. Lett. 106, 021301 (2011).

[13] E. Bergshoeff, O. Hohm, and P. Townsend, J. Phys. Conf. Ser. 229, 012005 (2010).

[14] S. Deser, Phys. Rev. Lett. 103, 101302 (2009).

[15] P. Nieuwenhuizen, Nucl. Phys. B60, 478 (1973).
[16] I. Antoniadis and E. Tomboulis, Phys. Rev. D 33, 2756 (1986).

[17] H. Nishino and S. Rajpoot, Phys. Lett. B 639, 110 (2006).

[18] A. Accioly, Phys. Rev. D 67, 127502 (2003).

[19] A. Accioly, Nucl. Phys. B, Proc. Suppl. 127, 100 (2004).

[20] A. Accioly and M. Dias, Int. J. Theor. Phys. 44, 1123 (2005).

[21] E. Bergshoeff, O. Hohm, and P. Townsend, Cosmology, the Quantum Vacuum and Zeta Functions: A Workshop with a Celebration of Emilio Elizalde's Sixtieth Birthday 2010, Bellaterra, Barcelona, Spain.

[22] A. Vilenkin, Phys. Rev. D 23, 852 (1981).

[23] A. Vilenkin and E. Shellard, Cosmic Strings and Other Topological Defects (Cambridge University Press, Cambridge, England, 2001).

[24] P. Teyssandier, Classical Quantum Gravity 6, 219 (1989).

[25] I. Gradshteyn and I. Ryzhic, Table of Integrals, Series, and Products (Academic Press, San Diego, 1994).

[26] L. Jacobs and C. Rebbi, Phys. Rev. B 19, 4486 (1979).

[27] A. Accioly and H. Blas, Phys. Rev. D 64, 067701 (2001).

[28] A. Accioly and H. Blas, Int. J. Theor. Phys. 43, 47 (2004).

[29] S. Deser, R. Jackiw, and S. Templeton, Phys. Rev. Lett. 48, 975 (1982); Ann. Phys. (N.Y.) 140, 372 (1982); 185, 406 (E) (1988). 